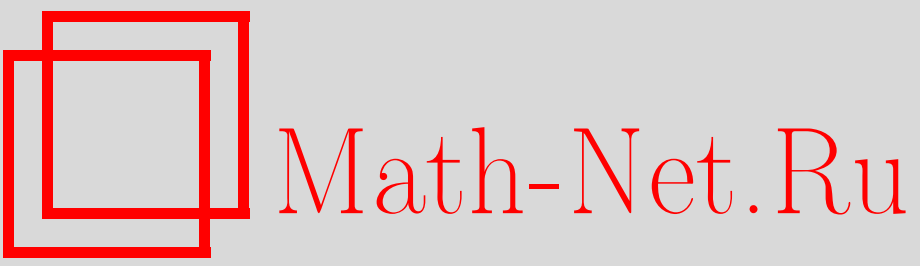

В. П. Радченко, Г. А. Павлова, С. В. Горбунов, Устойчивость по Ляпунову решений эндохронной теории пластичности без поверхности текучести в условиях плоского напряженного состояния, Вестн. Сам. гос. техн. ун-та. Сер. Физ.мат. науки, 2008, выпуск 2(), 143-151

DOI: https://doi.org/10.14498/vsgtu634

Использование Общероссийского математического портала Math-Net.Ru подразумевает, что вы прочитали и согласны с пользовательским соглашением http: //www . mathnet.ru/rus/agreement

Параметры загрузки:

IP : 35.173 .137 .237

26 апреля 2023 г., $11: 22: 23$ 


\title{
УДК 539.376;539.4;517.9 \\ УСТОЙЧИВОСТЬ ПО ЛЯПУНОВУ РЕШЕНИЙ ЭНДОХРОННОЙ ТЕОРИИ ПЛАСТИЧНОСТИ БЕЗ ПОВЕРХНОСТИ ТЕКУЧЕСТИ В УСЛОВИЯХ ПЛОСКОГО НАПРЯЖЕННОГО СОСТОЯНИЯ
}

\author{
В. П. Радченко, Г. А. Павлова, С. В. Горбунов
}

Самарский государственный технический университет,

443100, Самара, ул. Молодогвардейская, 244.

E-mail: radch@samgtu.ru

Предложсен вариант эндохронной теории высокотемпературной пластичности без поверхности текучести для разрушающегося материала. Исследована устойчивость решений по Ляпунову в условиях плоского напряжённого состояния. Построена предельная поверхность устойчивого дебормирования. Показано, что переход через эту поверхность связан с расходимостью численной итерационной процедуры расчёта напряжённо-деформированного состояния. Приведены примеры расчёта.

Ключевые слова: высокотемпературная пластичность, повреждённостъ материала, эндохронная теория, устойчивость решений по Ляпунову, предельная поверхность, расходимость итерационной процедуры.

Вопрос о построении определяющих соотношений высокотемпературной пластичности и в настоящее время остаётся открытым в силу ряда особенностей неупругого деформирования: зависимости диаграммы упругопластического деформирования от скорости деформирования, наличия деформации ползучести и других причин. В ряде работ, например, [1-3], предлагалось описывать ползучесть и пластичность металлов с единых позиций, при этом исходной информацией для построения определяющих соотношений пластичности является зависимость кривой «мгновенного» деформирования от скорости нагружения. Проблема предельных состояний пластически разрушающихся сред рассматривалась во многих работах как на уровне механики микронеоднородных сред [4-8], так и на феноменологическом уровне для макросреды $[8-10]$. В $[8,13]$ предложен эндохронный вариант теории пластичности с поверхностью текучести, описывающий для режима «жёсткого нагружения» и закритический (ниспадающий) участок диаграммы деформирования, а в [18] проанализирована проблема устойчивости решений этого варианта эндохронной теории пластичности в одноосном случае для режима «мягкого нагружения».

Целью настоящей работы является анализ устойчивости решений эндохронного варианта теории пластичности без поверхности текучести для случая плоского напряженного состояния для режима «мягкого нагружения».

Основные уравнения состояния предлагаемой модели в главных осях имеют вид:

Радченко Владимир Павлович - заведующий кафедрой прикладной математики и информатики Самарского государственного технического университета; д.ф.-м.н., профессор. Павлова Галина Александровна - дочент кафедры прикладной математики и информатики Самарского государственного технического университета; к.ф.-м.н, доцент. Горбунов Сергей Владимирович-студент Самарского государственного технического университета. 


$$
\begin{aligned}
& \varepsilon_{i}=e_{i}+e_{i}^{p}, \\
& e_{i}=\frac{1+\mu}{E} \sigma_{i}-\frac{\mu}{E}\left(\sigma_{1}+\sigma_{2}\right) \text {, } \\
& e_{i}^{p}=\frac{3}{2} \beta_{i}-\frac{1}{2}\left(\beta_{1}+\beta_{2}\right), \\
& \dot{\beta}_{i}=\left\{\begin{array}{cll}
\lambda\left[a S^{n-1} \sigma_{i}-\beta_{i}(t)\right], & \text { если } a S^{n-1} \sigma_{i}>\beta_{i}(t), \\
0, & \text { если } a S^{n-1} \sigma_{i} \leqslant \beta_{i}(t) ;
\end{array}\right. \\
& \sigma_{i}=\sigma_{i}^{0}(1+\omega), \\
& \dot{\omega}=\alpha\left(\sigma_{1} \dot{e}_{1}^{p}+\sigma_{2} \dot{e}_{2}^{p}\right), \\
& \beta_{i}(0)=0, \quad \omega(0)=0, \quad i=1,2 \text {, }
\end{aligned}
$$

где $e_{i}$ и $e_{i}^{p}$ - соответственно упругие и пластические деформации; $\beta_{i}$ - активные пластические деформации, которые можно было бы наблюдать в отсутствии пуассоновского сужения материала; $\sigma_{i}$ и $\sigma_{i}^{0}$ - истинные и номинальные напряжения; $\mu$ и $E$ - упругие постоянные; $c$ и $n$ - константы, описывающие участок упрочнения диаграммы пластического деформирования; $\alpha$ - параметр, контролирующий процессы пластического разупрочнения материала; $\lambda$ - параметр, характеризующий скорость нарастания пластической деформации; $\omega$-скалярный параметр поврежденности, который полагается пропорциональным работе истинных напряжений на пластических деформациях; $S=\frac{1}{\sqrt{2}} \sqrt{\left(\sigma_{1}-\sigma_{2}\right)^{2}+\sigma_{1}^{2}+\sigma_{2}^{2}}-$ интенсивность напряжений.

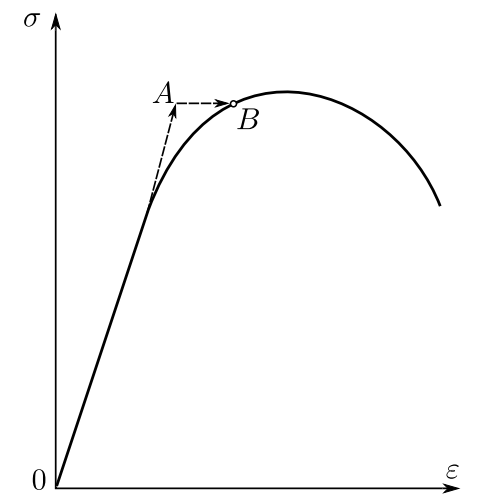

Рис. 1. Схема развития пластической деформации по эндохронной теории

Согласно соотношениям (3)-(4) пластическая деформация описывается такими же по структуре уравнениями, как и вязкопластическая компонента деформации ползучести $[8,13]$, т. е. также развивается во времени. Такой подход к описанию пластической деформации соответствует так называемым эндохронным теориям пластичности, т.е. теориям пластичности с внутренним временем. В предложенных уравнениях в качестве внутреннего времени используется и обычное физическое время. В частном случае одноосного напряжённого состояния схема развития упругопластической деформации в координатах $\sigma_{0} \sim \varepsilon$ в режиме мягкого нагружения (мгновенного приложения нагрузки) представляет ломаную $0 A B$ (см. рис. 1).

В уравнениях (3), (4) отсутствует поверхность текучести, т.е. деформации пластичности $e_{i}^{p}$ возникают при сколь угодно малых напряжениях. С одной стороны, это не противоречит современным физическим представлениям о том, что элементарные акты пластического деформирования в микрообъемах, особенно в условиях повышенных температур, могут происходить при очень малых напряжениях; с другой стороны, при малых значениях напряжения величина пластической деформации на несколько порядков меньше 
упругой составляющей (в силу нелинейности закона для пластической деформации и значительного значения величины $n$ в (4)) и при решении практических задач в условиях нестационарного нагружения никаких существенных погрешностей не внесет. Аналогичное утверждение можно найти и в [14]. Что же касается поверхности пластичности, разделяющей упругую разгрузку и активное пластическое деформирование, то её текущее состояние задаётся системой неравенств (4).

В [8] показано, что в режиме «жёсткого» нагружения $\left(\dot{e}^{p}=\right.$ const $)$ при одноосном нагружении уравнения (1)-(7) описывают полную диаграмму мгновенного деформирования, включая участок закритического деформирования. Целью настоящей работы является исследование решений системы уравнений (1)-(7) на устойчивость и определение границы устойчивого и неустойчивого деформирования при мягком нагружении в условиях плоского нагруженного состояния.

1. Вводя для удобства обозначения $e_{i}^{p}=x_{i}, \beta_{i}=y_{i}$, из (1)-(7) для схемы мягкого нагружения (заданы $\sigma_{1}^{0}$ и $\sigma_{2}^{0}$ ) получаем следующую задачу Коши:

$$
\begin{gathered}
\dot{y}_{i}=\lambda\left[a S^{n-1} \sigma_{i}-y_{i}(t)\right], \\
\sigma_{i}=\sigma_{i}^{0}(1+\omega), \\
\dot{\omega}=\alpha\left(\sigma_{1} \dot{x}_{1}+\sigma_{2} \dot{x}_{2}\right), \\
x_{i}=\frac{3}{2}\left(y_{i}-\frac{1}{2}\left(y_{1}+y_{2}\right)\right), \\
y_{i}(0)=0, \quad \omega(0)=0, \quad i=1,2 .
\end{gathered}
$$

Из уравнений (9)-(10) и начальных условий (12) можно получить первый интеграл системы:

$$
1+\omega=e^{\alpha\left(\sigma_{1}^{0} x_{1}+\sigma_{2}^{0} x_{2}\right)} .
$$

Учитывая (11), соотношение (13) можно записать в виде

$$
1+\omega=\exp \left[\alpha\left(y_{1}\left(\sigma_{1}^{0}-\frac{1}{2} \sigma_{2}^{0}\right)+y_{2}\left(\sigma_{2}^{0}-\frac{1}{2} \sigma_{1}^{0}\right)\right)\right] .
$$

Вводя обозначение

$$
T\left(y_{1}, y_{2}\right)=y_{1}\left(\sigma_{1}^{0}-\frac{1}{2} \sigma_{2}^{0}\right)+y_{2}\left(\sigma_{2}^{0}-\frac{1}{2} \sigma_{1}^{0}\right),
$$

перепишем (14) так:

$$
1+\omega=\exp \left[\alpha T\left(y_{1}, y_{2}\right)\right] .
$$

С учётом (9) и (15) величина интенсивности напряжений записывается в виде

$$
S=(1+\omega) \sqrt{\delta}=\sqrt{\delta} e^{\alpha T},
$$

где $\delta=\left(\sigma_{1}^{0}\right)^{2}-\sigma_{1}^{0} \sigma_{2}^{0}+\left(\sigma_{2}^{0}\right)^{2}$. 
Тогда (8) запишется следующим образом:

$$
\begin{aligned}
& \dot{y}_{1}=\lambda\left[a \sigma_{1}^{0} \delta^{\frac{n-1}{2}} e^{\alpha n T\left(y_{1}, y_{2}\right)}-y_{1}\right], \\
& \dot{y}_{2}=\lambda\left[a \sigma_{2}^{0} \delta^{\frac{n-1}{2}} e^{\alpha n T\left(y_{1}, y_{2}\right)}-y_{2}\right],
\end{aligned}
$$

где $T$ определяется по (15).

Итак, задача свелась к исследованию на устойчивость положения равновесия при различных комбинациях напряжений $\sigma_{1}^{0}$ и $\sigma_{2}^{0}$ системы уравнений (18).

Судя по виду системы (18), можно использовать метод Ляпунова исследования устойчивости по первому приближению [9]. Но сначала найдём положение равновесия. Приравниваем правые части (18) нулю:

$$
\left\{\begin{array}{l}
a \sigma_{0}^{1} \delta^{\frac{n-1}{2}} e^{\alpha n T\left(y_{1}, y_{2}\right)}-y_{1}=0 \\
a \sigma_{0}^{2} \delta^{\frac{n-1}{2}} e^{\alpha n T\left(y_{1}, y_{2}\right)}-y_{2}=0
\end{array}\right.
$$

откуда

$$
y_{1}=\frac{\sigma_{1}^{0}}{\sigma_{2}^{0}} y_{2}
$$

Тогда для величины $T$ имеем:

$$
T\left(y_{1}, y_{2}\right)=\left(\frac{\sigma_{1}^{0}}{\sigma_{2}^{0}}\left(\sigma_{1}^{0}-\frac{1}{2} \sigma_{2}^{0}\right)+\sigma_{2}^{0}-\frac{1}{2} \sigma_{1}^{0}\right) y_{2}=\frac{y_{2}}{\sigma_{2}^{0}} \delta .
$$

Подставляя значение $T$ во второе уравнение (19), получим уравнение относительно $y_{2}$ :

$$
y_{2}=a \sigma_{2}^{0} \delta^{\frac{n-1}{2}} \exp \left(\frac{n \alpha \delta}{\sigma_{2}^{0}} y_{2}\right)
$$

которое при заданных $\sigma_{1}^{0}$ и $\sigma_{2}^{0}$ может быть решено численно.

Обозначая корень $(21)$ через $y_{2}^{0}$, из $(20)$ найдём $y_{1}^{0}$. Таким образом, получаем положение равновесия $\left(y_{1}^{0}, y_{2}^{0}\right)$.

Введём возмущенные переменные:

$$
u=y_{1}-y_{1}^{0}, \quad v=y_{2}-y_{2}^{0} .
$$

Тогда $\dot{u}=\dot{y}_{1}, \dot{v}=\dot{y}_{2}$, и для величины $T$ из (15) имеем:

$$
\begin{aligned}
& T\left(y_{1}, y_{2}\right)=\left(u+y_{1}^{0}\right)\left(\sigma_{1}^{0}-\frac{1}{2} \sigma_{2}^{0}\right)+\left(v+y_{2}^{0}\right)\left(\sigma_{2}^{0}-\frac{1}{2} \sigma_{1}^{0}\right)= \\
& =u\left(\sigma_{1}^{0}-\frac{1}{2} \sigma_{2}^{0}\right)+v\left(\sigma_{2}^{0}-\frac{1}{2} \sigma_{1}^{0}\right)+y_{1}^{0}\left(\sigma_{1}^{0}-\frac{1}{2} \sigma_{2}^{0}\right)+y_{2}^{0}\left(\sigma_{2}^{0}-\frac{1}{2} \sigma_{1}^{0}\right)= \\
& =T(u, v)+T\left(y_{1}^{0}, y_{2}^{0}\right)=T(u, v)+T_{0},
\end{aligned}
$$

где $T_{0}=T\left(y_{1}^{0}, y_{2}^{0}\right)$

146 
В результате получаем новую систему, положение равновесия которой $u=0, v=0$ исследуем на устойчивость с помощью системы по первому приближению:

$$
\left\{\begin{array}{l}
\dot{u}=f_{1}(u, v) \\
\dot{v}=f_{2}(u, v)
\end{array}\right.
$$

где

$$
\begin{aligned}
& f_{1}(u, v)=\lambda\left[a \sigma_{1}^{0} \delta^{\frac{n-1}{2}} \exp \left[n \alpha\left(T(u, v)+T_{0}\right)\right)-u-y_{1}^{0}\right] \\
& f_{2}(u, v)=\lambda\left[a \sigma_{2}^{0} \delta^{\frac{n-1}{2}} \exp \left[n \alpha\left(T(u, v)+T_{0}\right)\right]-v-y_{2}^{0}\right]
\end{aligned}
$$

Составляем матрицу Якоби:

$$
\begin{gathered}
A=\left.\left(\begin{array}{ll}
\frac{\partial f_{1}}{\partial u} & \frac{\partial f_{1}}{\partial v} \\
\frac{\partial f_{2}}{\partial u} & \frac{\partial f_{2}}{\partial v}
\end{array}\right)\right|_{\substack{u=0 \\
v=0}}= \\
=\left[\begin{array}{cc}
\lambda\left(a \sigma_{1}^{0} \delta^{\frac{n-1}{2}} e^{n \alpha T_{0}} n \alpha\left(\sigma_{1}^{0}-\frac{1}{2} \sigma_{2}^{0}\right)-1\right) & \lambda a \sigma_{1}^{0} \delta^{\frac{n-1}{2}} e^{n \alpha T_{0}} n \alpha\left(\sigma_{2}^{0}-\frac{1}{2} \sigma_{1}^{0}\right) \\
\lambda a \sigma_{2}^{0} \delta^{\frac{n-1}{2}} e^{n \alpha T_{0}} n \alpha\left(\sigma_{1}^{0}-\frac{1}{2} \sigma_{2}^{0}\right) & \lambda\left(a \sigma_{2}^{0} \delta^{\frac{n-1}{2}} e^{n \alpha T_{0}} n \alpha\left(\sigma_{2}^{0}-\frac{1}{2} \sigma_{1}^{0}\right)-1\right)
\end{array}\right] .
\end{gathered}
$$

Обозначив $\beta=a \delta^{\frac{n-1}{2}} n \alpha \exp \left(n \alpha T_{0}\right), \sigma_{1}^{*}=\sigma_{1}^{0}-\frac{1}{2} \sigma_{2}^{0}, \sigma_{2}^{*}=\sigma_{2}^{0}-\frac{1}{2} \sigma_{1}^{0}$, находим характеристические числа $r_{1}$ и $r_{2}$ этой матрицы из уравнения $\operatorname{det}(A-r E)=0$ :

$$
\left|\begin{array}{cc}
\lambda\left(\beta \sigma_{1}^{0} \sigma_{1}^{*}-1\right)-r & \lambda \beta \sigma_{1}^{0} \sigma_{2}^{*} \\
\lambda \beta \sigma_{2}^{0} \sigma_{1}^{*} & \lambda\left(\beta \sigma_{2}^{0} \sigma_{2}^{*}-1\right)-r
\end{array}\right|=0,
$$

откуда получаем

$$
r^{2}-r \lambda\left(\beta\left(\sigma_{1}^{0} \sigma_{1}^{*}+\sigma_{2}^{0} \sigma_{2}^{*}\right)-1\right)-\lambda^{2}\left(\beta\left(\sigma_{1}^{0} \sigma_{1}^{*}+\sigma_{2}^{0} \sigma_{2}^{*}\right)-1\right)=0
$$

Учитывая

$$
\sigma_{1}^{0} \sigma_{1}^{*}+\sigma_{2}^{0} \sigma_{2}^{*}=\sigma_{1}^{0}\left(\sigma_{1}^{0}-\frac{1}{2} \sigma_{2}^{0}\right)+\sigma_{2}\left(\sigma_{2}^{0}-\frac{1}{2} \sigma_{1}^{0}\right)=\sigma_{1}^{02}-\sigma_{1}^{0} \sigma_{2}^{0}+\sigma_{0}^{2}=\delta
$$

имеем характеристическое уравнение системы:

$$
r^{2}+a_{1} r+a_{2}=0
$$

где $a_{1}=-\lambda(\beta \delta-1), a_{2}=-\lambda^{2}(\beta \delta-1)$.

Для двумерного случая необходимым и достаточным условием асимптотической устойчивости положения равновесия является условие А. Стодолы $[15]: a_{1}>0, a_{2}>0$, т. е.

$$
\left\{\begin{array}{l}
-\lambda(\beta \delta-1)>0 \\
-\lambda^{2}(\beta \delta-1)>0
\end{array}\right.
$$

откуда

$$
\beta \delta-1<0
$$


Расписывая (23) более подробно, получаем условие асимптотической устойчивости положения равновесия в виде

$$
a \delta^{\frac{n+1}{2}} e^{n \alpha T_{0}} n \alpha<1,
$$

где $\delta=\left(\sigma_{1}^{0}\right)^{2}-\sigma_{1}^{0} \sigma_{2}^{0}+\left(\sigma_{2}^{0}\right)^{2}, T_{0}=y_{1}^{0}\left(\sigma_{1}^{0}-\frac{1}{2} \sigma_{2}^{0}\right)+y_{2}^{0}\left(\sigma_{2}^{0}-\frac{1}{2} \sigma_{1}^{0}\right)$, при этом $\left(y_{1}^{0}, y_{2}^{0}\right)$ - положение равновесия, соответствующее паре напряжений $\left(\sigma_{1}^{0}, \sigma_{2}^{0}\right)$, находится численно по (20), (21).

Вводя обозначение

$$
k\left(\sigma_{1}^{0}, \sigma_{2}^{0}\right)=a \delta^{\frac{n+1}{2}} e^{n \alpha T_{0}} n \alpha,
$$

прокомментируем полученные результаты с точки зрения механики деформируемого твердого тела. Если мы имеем схему мягкого нагружения, то согласно модели (см. рис. 1) формально сначала решается упругая задача, а затем рассчитывается пластическая деформация, которая развивается во времени (расчёт ведётся по аналогии с деформацией ползучести). Здесь возможны следующие ситуации:

- если пара номинальных напряжений $\left(\sigma_{1}^{0}, \sigma_{2}^{0}\right)$ удовлетворяет условию $k\left(\sigma_{1}^{0}, \sigma_{2}^{0}\right)<1$, то компоненты тензора пластических деформаций $e_{1}^{p}$ и $e_{2}^{p}$ при $t \rightarrow \infty$ стремятся к своим асимптотическим значениям, которые и принимаются за величины пластических деформаций, при этом при $k\left(\sigma_{1}^{0}, \sigma_{2}^{0}\right)=1$ имеем множество точек $\left(\sigma_{1}^{0}, \sigma_{2}^{0}\right)$, отделяющих область асимптотической устойчивости решений системы (1)-(7) от неустойчивой области; другими словами, плоская кривая $k\left(\sigma_{1}^{0}, \sigma_{2}^{0}\right)=1$ - это множество предельных состояний материала при плоском напряжённом состоянии;

- если $k\left(\sigma_{1}^{0}, \sigma_{2}^{0}\right)>1$, то с точки зрения механики после решения упругой задачи в силу расходимости решений (1)-(7) имеем $\lim _{t \rightarrow \infty} e_{i}^{p}(t)=\infty$, и это означает, что в реальном эксперименте это состояние достигнуто быть не может.

2. Рассмотрим модельный расчёт для сплава ЭИ 698 при $T=750^{\circ} \mathrm{C}$.

\begin{tabular}{|c|c|c|c|}
\hline \multicolumn{4}{|c|}{$\begin{array}{c}\text { Значения параметров модели для описания деформац } \\
\text { пластичности сплава ЭИ698 при } T=750^{\circ} \mathrm{C}\end{array}$} \\
\hline$E \cdot 10^{-5}, \mathrm{MПа}$ & $n$ & $a,(\mathrm{M \Pi \textrm {a }})^{-n}$ & $\alpha,(\mathrm{M \Pi а})^{-1}$ \\
\hline 1,5 & 7,99 & $9,52 \cdot 10^{-26}$ & $2,74 \cdot 10^{-3}$ \\
\hline
\end{tabular}
Характеристики модели (1)-(7) представлены в таблице.

На рис. 2 представлена экспериментальная и расчётная диаграммы деформирования этого сплава в одноосном случае $\left(\sigma_{2}^{0}=0\right)$ по схеме мягкого нагружения. В данном случае критическое значение $k=1$ достигается при $\sigma_{1}^{0}=870 \mathrm{MПа,} \mathrm{что} \mathrm{хорошо} \mathrm{коррелирует,} \mathrm{например,} \mathrm{с} \mathrm{экспериментальным}$ значением временного предела сопротивления $\sigma_{\text {вр }}=858$ МПа. Штриховая линия на рис. 2 подчёркивает, что в предельном случае расчётная пластическая деформация становится бесконечно большой. 

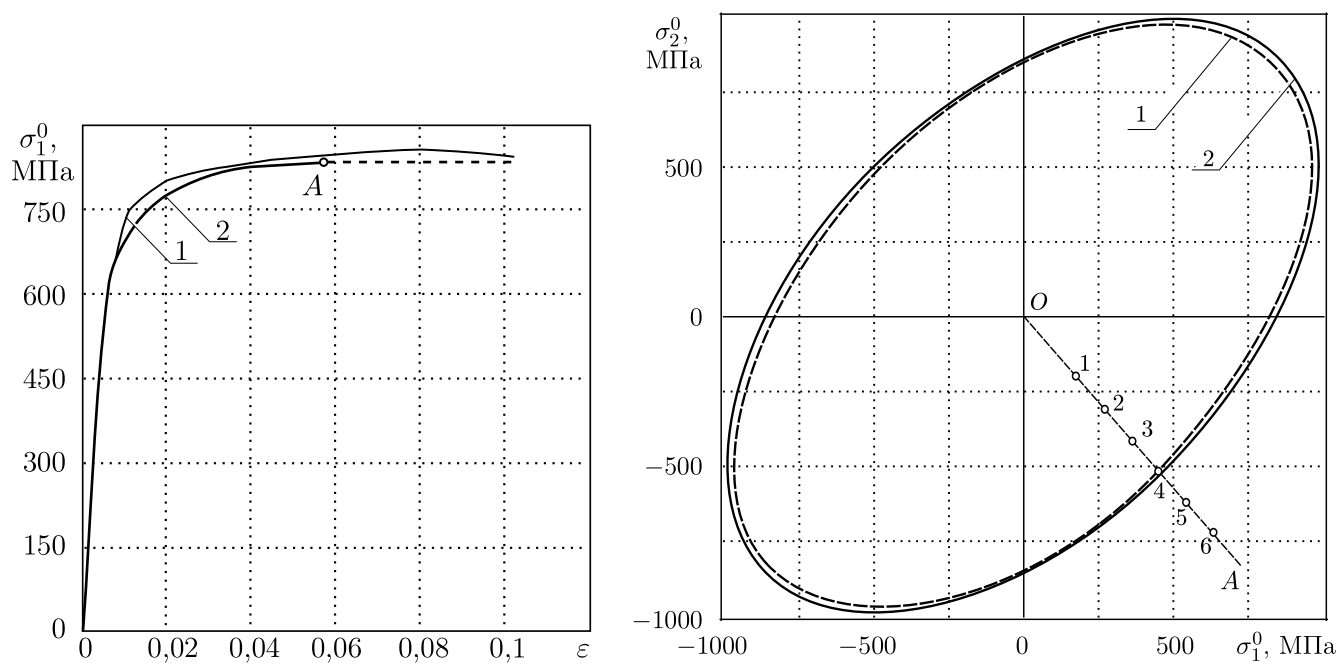

Рис. 2. Экспериментальная (2) и рас- Рис. 3. Предельные поверхности устойчичётная (1) диаграммы деформирова- вого деформирования по методу Ляпунония сплава ЭИ 698 при $T=750^{\circ} \mathrm{C}$ ва (2) и методу Эйлера (1)

На рис. 3 приведена расчётная предельная зависимость для сплава ЭИ 698 при $T=750^{\circ} \mathrm{C}$ при плоском напряженном состоянии, соответствующая условию $k\left(\sigma_{1}^{0}, \sigma_{2}^{0}\right)=1$. Внутри области имеем асимптотически устойчивые режимы деформирования $(k<1)$, а вне её $(k>1)$ - неустойчивые режимы (с математической точки зрения), которые физически для режима мягкого нагружения реализованы быть не могут.

3. Для выявления характера неустойчивости решения системы (1)-(7) при $k\left(\sigma_{1}^{0}, \sigma_{2}^{0}\right)>1$ выполнено численное решение этой системы дифференциальных уравнений по методу Эйлера, и оказалось, что при переходе через значения $\left(\sigma_{1}^{0}, \sigma_{2}^{0}\right)$, удовлетворяющие (в рамках вычислительной погрешности) условию $k\left(\sigma_{1}^{0}, \sigma_{2}^{0}\right)=1$, качественно меняется характер кривых деформиро-
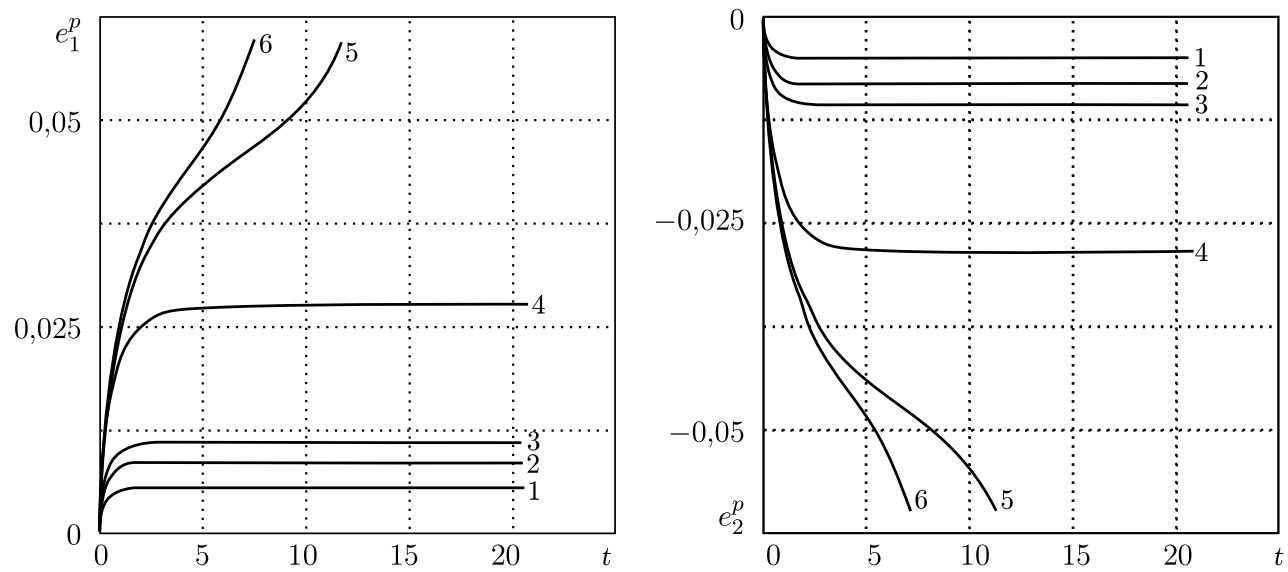

Рис. 4. Кривые деформирования «пластическая деформация - внутреннее время».

Цифры 1-6 соответствуют напряжённым состояниям на луче $O A$ (см. рис. 3) 
вания «пластическая деформация - внутреннее время». В качестве примера на рис. 4 показаны расчётные зависимости $e_{i}^{p}(t)$ при различных сочетаниях $\left(\sigma_{1}^{0}, \sigma_{2}^{0}\right)$ на луче $O A$. Если проводить аналогию с кривыми ползучести, то при $k<1$ эти кривые асимптотически ограничены при $t \rightarrow \infty$, а при $k>1$ на этих кривых появляется третья (ускоренная) стадия и $\lim _{t \rightarrow \infty} e_{i}^{p}=\infty$, т. е. всем значениям $\left(\sigma_{1}^{0}, \sigma_{2}^{0}\right)$ в этой области соответствует расходящийся во времени вычислительный процесс и бесконечное значение компонент тензора деформаций пластичности. В качестве примера на рис. 3 показаны границы устойчивого деформирования по критерию $k\left(\sigma_{1}^{0}, \sigma_{2}^{0}\right)=1$ и расходимости численной процедуры метода Эйлера. Отсюда можно сделать вывод о коррелированности условия устойчивости решений системы (1)-(7) по Ляпунову и расходимости вычислительной процедуры метода Эйлера. Аналогичный результат отмечался в работах В. В. Стружанова при исследовании закритического упругопластического деформирования $[10,16]$.

Таким образом, предельная зависимость $k\left(\sigma_{1}^{0}, \sigma_{2}^{0}\right)=1$ устойчивости системы (1)-(7) по Ляпунову можно трактовать как границу устойчивого упругопластического деформирования и перехода на закритическую стадию.

\section{БИБЛИОГРАФИЧЕСКИЙ СПИСОК}

1. Кадашевич, Ю.И. Теория пластичности и ползучести, учитывающая наследственные свойства и влияние скорости пластического деформирования на локальный предел текучести материала [Текст] / Ю. И. Кадашевич, В.В.Новожилов // Докл. АН СССР. 1978. - Т. 238. - № 1. - С. 36-38.

2. Клебанов, Я. М. Методика определения параметров неупругого реономного деформирования [Текст] / Я. М. Клебанов, И. А. Кокорев // Заводская лаборатория. - 1985. № 4. - C. $80-83$.

3. Садаков, О. С. Анализ напряженно-деформированного состояния элементов конструкций на основе структурной модели среды [Текст] / О. С. Садаков // Материалы Всесоюзного симпозиума по малоцикловой усталости при повышенных температурах. Челябинск. - 1974. - Вып. 3. - С. 95-127.

4. Кадашевич, Ю. И. Расширенный вариант теории ползучести, учитывающий микроразрушения [Текст] / Ю.И. Кадашевич, А. М. Пейсахов, С. П. Помыткин // Вестн. Сам. гос. техн. ун-та. Сер. Физ.-мат. науки. - 2008. - № 1(16). - С. 33-35.

5. Кадашевич, Ю. И. Теория пластичности и ползучести, учитывающая микроразрушения [Текст] / Ю. И. Кадашевич // Докл. АН СССР. - 1982. - Т. 266. — № 6. - С. 79-87.

6. Новожилов, В. В. Микронапряжения в конструкционных материалах [Текст] / В.В.Новожилов, Ю. И. Кадашевич. - Л.: Машиностроение, 1990. - 224 с.

7. Стружанов, В. В. Модификационная модель Мизинга [Текст] / В. В. Стружанов, Вяч. В. Башуров // Вестн. Сам. гос. техн. ун-та. Сер. Физ.-мат. науки. - 2007. — № 1(14). C. 20-39.

8. Радченко, В. П. Реологическое деформирование и разрушение материалов и элементов конструкций [Текст] / В. П. Радченко, Ю. А. Еремин. - М.: Машиностроение-1, 2004. 265 с. - ISBN 5-94275-111-0.

9. Стружанов, В.В. Свойства разупрочняющихся материалов и определяющие соотношения при одноосном напряженном состоянии[Текст] / В. В. Стружанов // Вестн. Сам. гос. техн. ун-та. Сер. Физ.-мат. науки. - 2007. — № 2(15). - С. 69-78.

10. Стружанов, В. В. Деформационное разупрочнение материала в элементах конструкций [Текст] / В. В. Стружанов, В. И. Миронов. - Екатеринбург: УрО РАН, 1995. - 191 с.

11. Радченко, В. П. Математическая модель неупругого деформирования и разрушения металлов при ползучести энергетического типа [Текст] / В.П. Радченко // Вестн. Сам. гос. техн. ун-та. Сер. Физ.-мат. науки. - 1996. - № 4. - С. 43-63.

12. Радченко, В.П. Об устойчивости решений одного варианта эндохронной теории одноосной пластичности [Текст] / В.П. Радченко, Г. А. Павлова, С. В. Горбунов / Матем. моделирование и краев. задачи: Тр. Пятой Всерос. научн. конф. с междунар. участи- 
ем. - Самара: СамГТУ, 2008. - Ч.1: Математические модели механики, прочности и надёжности элементов конструкций. - С. 255-261.

13. Самарин, Ю. П. Уравнения состояния материалов со сложными реологическими свойствами [Текст] / Ю. П. Самарин. - Куйбышев: Куйбышевский госуниверситет, 1979. $84 \mathrm{c}$.

14. Соснин, О.В. О термопластичности [Текст] / О.В. Соснин, О.О. Соснин // Пробл. прочности. - 1988. - № 12. - С. 3-9.

15. Меркин, Д. Р. Теория устойчивости в примерах и задачах [Текст] / Д. Р. Меркин, С. М. Бауэр, А.Л. Смирнов, Б. А. Смольников. - М.-Ижевск: НИЦ «Регулярная и хаотическая динамика», Ин-т компьютерных исследований, 2007. - 208 с.

16. Жижерин, C.В. Итерационные методы расчёта напряжений при чистом изгибе балки из повреждаемого материала [Текст] / С. В. Жижерин, В. В. Стружанов, В. И. Миронов // Вычислительные технологии. - 2001. - Т. 6. - № 5. - С. 24-33.

Поступила в редакцию 05/VII/2008;

в окончательном варианте - 12/X/2008.

MSC: $74 \mathrm{Cxx}, 74 \mathrm{G} 55$

\section{STABILITY BY LYAPUNOV OF SOLUTIONS IN ENDOCHRONIC PLASTICITY THEORY WITHOUT FLUIDITY SURFACE IN FLAT TENSION CONDITIONS}

\section{V.P. Radchenko, G. A. Pavlova, S. V. Gorbunov}

Samara State Technical University,

443100, Samara, Molodogvardeyskaya str., 244.

E-mail: radch@samgtu.ru

The variant of endochronic theory of high-temperature plasticity without fluidity surface for a collapsing material is studied. Stability by Lyapunov solutions in flat tension conditions is investigated. The limiting surface of steady deformation is constructed. It is shown that transition through this surface correlates with divergence of numerical iterative calculation procedure. Calculation examples are quoted.

Key words: high-temperature plasticity, damage of material, the endochronic theory, Lyapunov stability of solutions, a limiting surface, divergence of iterative procedure.

Original article submitted 05/VII/2008; revision submitted $12 / \mathrm{X} / 2008$.

Radchenko Vladimir Pavlovich, Dr. Sci. (Phis. E Math.), Prof., Head of Dept. of Applied Mathematics and Computer Science of Samara State Technical University.

Pavlova Galina Alexandrovna, Ph.D. (Phis. ES Math.) Assist. Prof., Dept. of Applied Mathematics and Computer Science of Samara State Technical University.

Gorbunov Sergey Vladimirovich, Student of Samara State Technical University. 